\title{
Measuring compactness of the urban landscape within a city territory for environmental capabilities: The case of 50 cities in Eastern China
}

\author{
Chen S. Shuang ${ }^{1}$, Zhang Tong ${ }^{12}$, Li Guangyu ${ }^{3}$, Yan Yue ${ }^{4}$ \\ ${ }^{1}$ Nanjing Institute of Geography \& Limnology, Chinese Academy Sciences, China ${ }^{2}$ Graduate \\ University of Chinese Academy of Sciences, Beijing, China ${ }^{3}$ Nanjing Institute of Environmental \\ Sciences, Ministry of Environmental Protection, Nanjing, China ${ }^{4}$ Shandong Urban and Rural \\ Planning Design Institute, Jinan, China \\ E-mail: schens@niglas.ac.cn, zhangtongrose@163.com, leeguangyu@126.com,yyue_666@163. \\ com
}

\begin{abstract}
When a compact city is pursued as the strategy for urban sustainability the understanding of compactness is varied from the developed countries to the developing countries. In China the historical cities are characterized with high density and mixed land use. After a short time of motorization they still show compact forms in the central city. A large amount of newly developed areas are distributed in clusters near or far from the center. The crop land and natural habitat are encroached to different degrees. This paper developed an approach to measure the structural compactness of urban landscape patches within a city territory. It included six spatial metrics to measure the shape and density of the central agglomeration, the area configuration and distant relationship between the central agglomeration and the other clusters, and the distribution of all urban patches. By this approach the 50 cities in eastern China were categorized into five classes of forms: mono-center, multi-nucleus, centralized group, cluster group and scatter. Then the vegetation biomass loss with urban expansion was calculated based on remote sensing data, and used to assess the environmental capability of the five types of urban form. The suggestions of urban form optimization could be put forward for the five categories of cities.
\end{abstract}

Keywords: Compactness, Chinese city, spatial metrics, vegetation biomass, urban planning

\section{Introduction}

Under the background of rapid urbanization worldwide, a compact city is promoted to mitigate the adverse consequences of urban area growth. It features a high density and mixed-use urban form, being claimed to conserve arable land and wildlife habitats, decrease travel by car and reduce fuel emission, improve access to services and facilities and provide more efficient public infrastructure. Despite the policy in land use policy and urban planning in many countries, the meaning of compactness lacks of consensus and few data are sought to support the claimed advantages (Jenks, Burton \& Williams, 1996). Thus different indicators are studied to measure the variety of attributes of compactness. For instance, Burton (2002) defined a set of indicators at dimensions of density, mixeduse and intensity and tested in 25 UK towns. These cities performed differently for different indicators, and the scores for individual rather than the composite indicators of compactness display greater contrasts between cities. This divergent result is clearly not conductive to a clear description of traits of compactness, upon which the causes and consequences 
are to be studied. Considering uncertainty in and huge demand for the data of population density and mixed land use, morphological measures are much feasible to describe the actual physical form of built-up areas with the clarified definition, detailed and robust spatial data. A range of spatial metrics derived from the definition of sprawl condition, have been adopted to measure urban compactness as the other side of the coin (Galster et al., 2001; Tsai, 2005). They depicting land use patterns at dimensions of density, continuity, centrality, clustering etc., can be compared among cities in different countries (Angel et al., 2005; Huang et al., 2007; Hagen-Zanker \& Timmermans, 2008). In addition the physical and spatial pattern of urban areas provides a basis upon which to achieve urban efficiency. For instance, through selecting particular urban form we can estimate how much energy can be saved. To build such a consistent planning paradigm there are still some lack of studies aimed at integrated innovative analytical approach and accurate assessment of urban performance under investigation (Silva, 2016). This paper will take the real case scenarios of Chinese cities to study the physical forms of built-up areas aiming at assessing urban performance. Chinese cities have two thousand years of history, many of the old city developed within the city wall until the 1950s. Since the 1980s, the process of urbanization accelerated in the form of increase in the number and scale. The new city was built on the basis of the original county town. Big cities, in addition to their own outward extension, but also built some secondary centers in their territories through the establishment of development zones, new towns, and etc., thus promoting the expansion of urban scale. During this period, the population growth and employment needs drove urban expansion on the hand of endogenous stimuli, and on the other hand the urban planning regulated to a large extent the scale and morphological changes of Chinese cities (Chen et al., 2009). China's urban planning policy which took the excessive expansion of the city in the West and other developing countries as a mirror, strictly restricted the development of large cities. In the perspective of saving land, a criterion of per square kilometer per 10,000 people has been severely implemented in the master urban plan, effectively maintaining the high population density in the central city. At the same time, the group development is promoted to meet the needs of urban growth. The group layout of compact urban clusters in a city territory has been reflected in the master plan. In this context, most of the city's central area and other concentration areas are dense, although it cannot be said that the full efficient use of land, the study of compact development shifts to focus on the compactness of city group structure (Fang \& Qi, 2007; Ma \& Jin, 2011). It involves not only the centralized layout of edge growth to a core but also the multicenter concentration, or multi-level center of the centralized layout. Thus the dimension of global spatial autocorrelation should be considered to reflect the compact thinking of the group.

\section{Methodology}

Considering the multi-dimensional and multiindex characteristics of urban compactness, a clustering analysis method was used in this study, by which the cities with significant discrepancy in compactness were quantitatively differentiated and classified. Based on the SPSS19.0 software, the Euclidean metric is chosen as the measure of similarity, and according to the values of compactness index the sample cities are classified by the Wards method of hierarchical clustering. On the one hand, the classification approach can partly keep information of the basic indicators and avoid the semantic ambiguity of the composite index. On the other hand, the synthetic type through classification can be used as the basis upon which urban performance be assessed, and effect index by types can be obtained and discussed in association with compactness. In this study the indicator of vegetation biomass loss under urban expansion is adopted for the performance assessment. A higher indicator value denotes the bigger ecological loss caused by urban expansion in the selected urban form type, meaning the effects of the compactness type is less preferred.

The spatial metrics are selected to reflect the 
structural compactness. Because of the coreperiphery structure of Chinese cities all the urban clusters in a city territory are separated into the central built-up area and the others. The dimensions of density, shape, distance and spatial autocorrelation have been considered for the individual or composite characteristics of the two types of urban clusters. The density and shape measures are only applied to the central built-up area which hold the major urban activities, as the first level of compactness metrics. The second level of compactness measures reflect the spatial structure of the central area and the other urban landscape patches, including their area composition and distance distribution. In addition, the global spatial autocorrelation index is used to analyze the spatial layout characteristics of all patches as the third level of compactness measure. Each level of specific indicators are chosen from the defined spatial metrics in literature (Richardson, 1973; Brueckner \& Fansler, 1983; Cole, 1964; Tsai, 2005). After repeated comparison of index feasibility, applicability in Chinese cities and data availability, six indicators have been determined in this study, including the Richardson compactness, density gradient at the first level; continuity, Galster centrality at the second level; Gini coefficient and Moran'I coefficient at the third level. Their definition and calculation methods are shown in Table 1.

The study area is located in the central eastern coastal area of China, including all the designated cities in the Yangtze River Delta excepting for Zhoushan which is composed of islands. The total of 50 selected cities include two types: the city with district (or prefecture-level city) and the city without district (or county-level city). The territory of the first type city is the outline boundary of its urban districts, excluding the area under jurisdiction of the county, for the second type city the territory is the administrative scope (Fig. 1). In this study the built-up areas in a city territory involves the central one and its peripheral clusters, key towns, not including rural settlements. We obtain the patch vector data of the built areas from interpretation of the satellite images with the resolution of 30 meters in 2000 and 2010, which is used for calculation of the spatial metrics. Moreover the data of the urban land within the concentric circles for the density gradient calculation, as well as the built-up area on the grid for spatial autocorrelation analysis can be acquired by the superposition operation of the vector data.

Taking vegetation biomass as an example, this study attempts to assess the city performance with different compactness. Vegetation biomass presenting the total amount of organic matter (dry weight) in a unit area, at a given time, and distinguishing the types of green areas quantitatively, is mapped based on multi-sourced data, including remote sensing, meteorology, land use/cover, forest inventory, and grain yield (Li et al., 2016). With the data of built-up area change from 2000 to 2010, the biomass loss values per unit area of urban expansion is calculated for the assessment. The higher the value the lower the benefit of the compact city type in a view of vegetation conservation.

\section{Measurement and analysis}

Results for the six compactness metrics are reported for each of the 50 YRD cities. Similar to the previous literature, different indicators perform different among the cities by the bar graph (Fig. 2). When considering the size of the city the large cities are characterized with some lower density gradient and higher Moran'I, while variation of the indicator among small cities is little. In general the indicators and the size of the city is not particularly consistent with the law of change, revealing that the indicators of independence is strong. Five urban form types are obtained by cluster analysis. For each type, the difference between the internal mean value and the mean value of each index is expressed by standard deviation (Table 2). When the average of one or more indicators within a type is much higher than the mean of corresponding indicator, this type is regarded to be dominated by this or several indicators. According to the core-peripheral relations denoted by the indicators, combined with the experience observation of real case of urban areas, the five types are defined and modeled, 


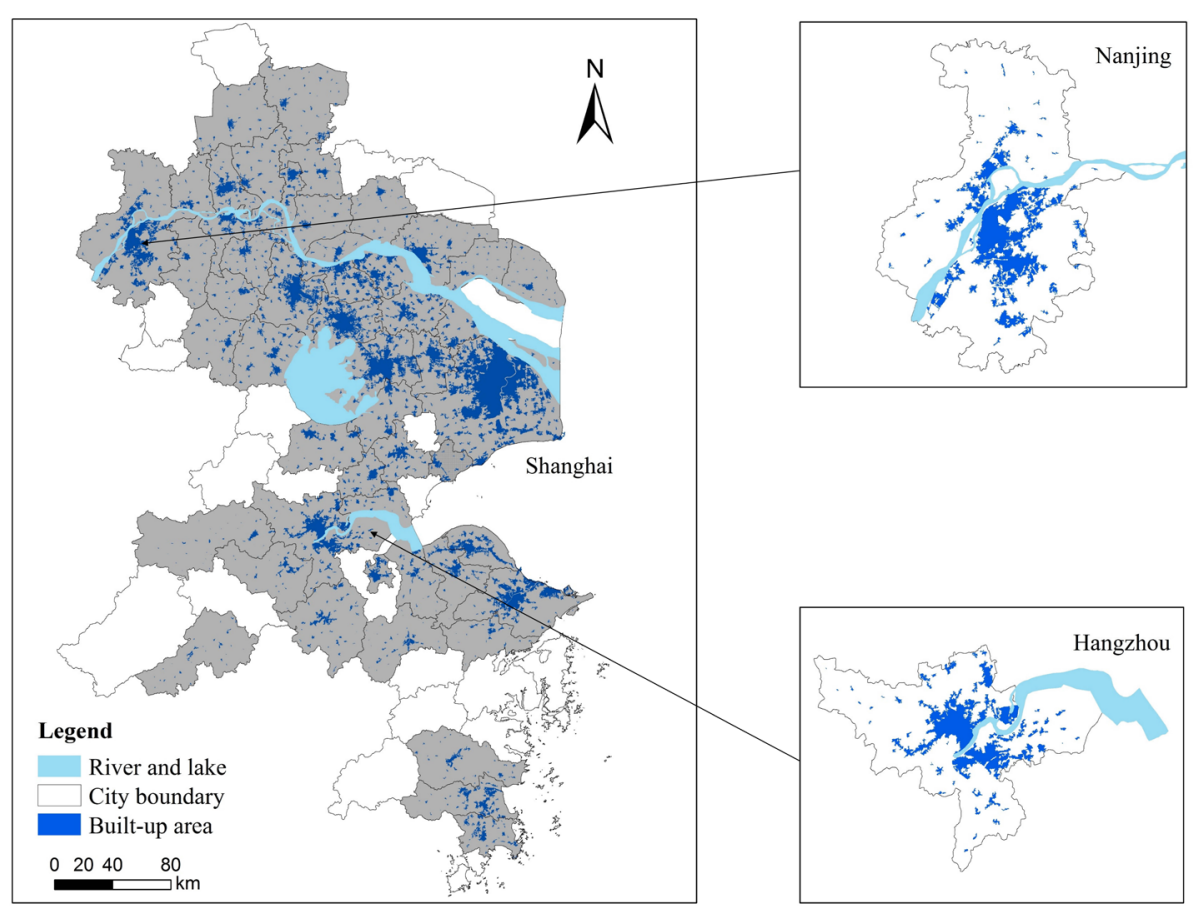

Figure 1. Location of the 50 cities in the Yangtze River Delta region in eastern China
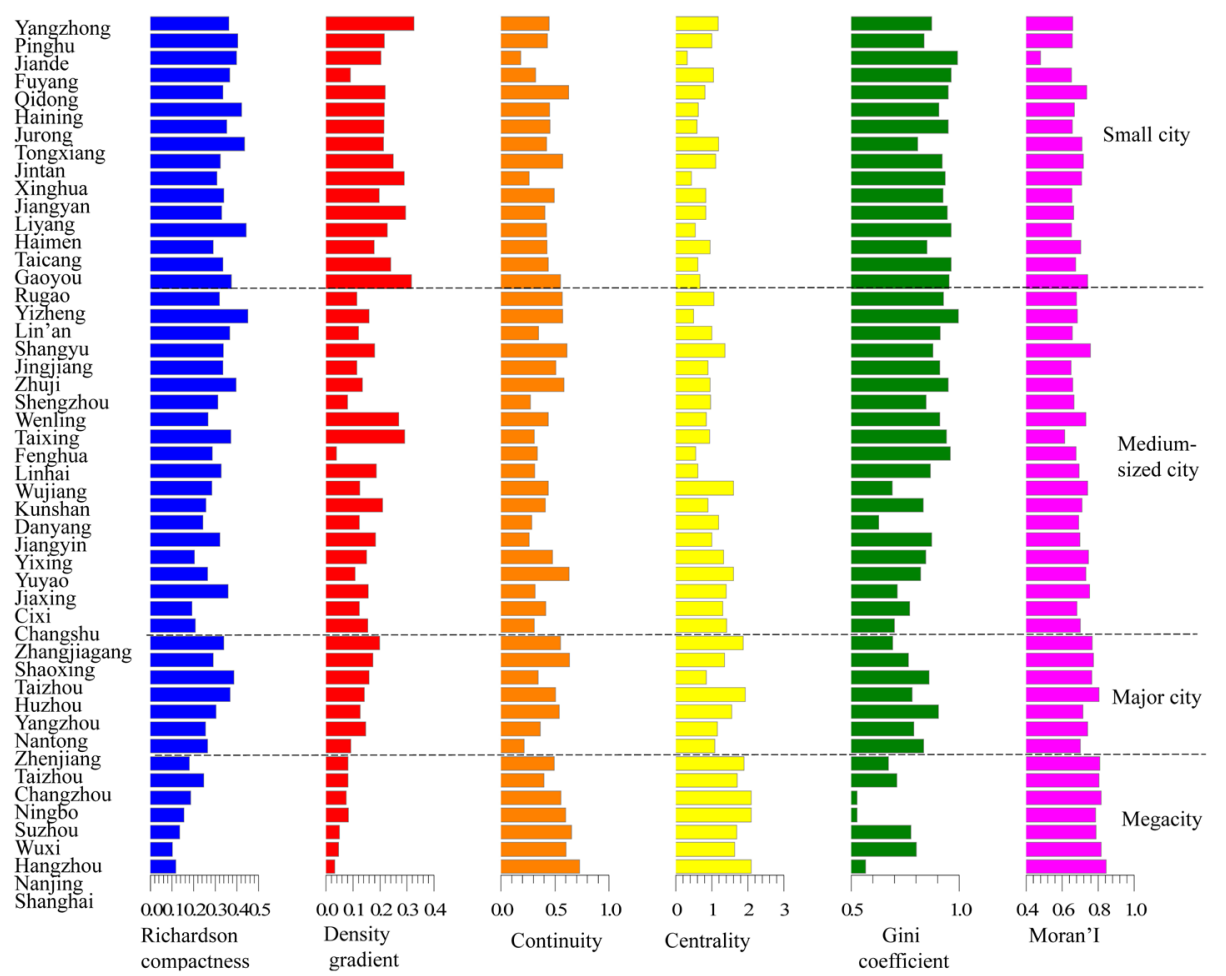

Figure 2. Variation of $\mathbf{6}$ spatial metrics among 50 cities in the Yangtze River Delta region Note: according to statistical yearbook of 2009 from National Bureau of Statistics of China, 50 cities are divided into four categories: megacity (more than 1 million people), major city (0.5 1 million people), medium-sized city (0.2 0.5 million people), small city (less than 0.2 million people) 
such as the type of centralized group, the type of scattering and so on (Fig. 3). The details are illustrated in the following paragraphs.

Type I involves the cities in the form of centralized group, including Shanghai, Nanjing, Wuxi, Hangzhou and Suzhou. For the five cities the continuity, centrality and Moran' I index are higher than those of other categories, indicating that the urban land patches in the city territory is balanced
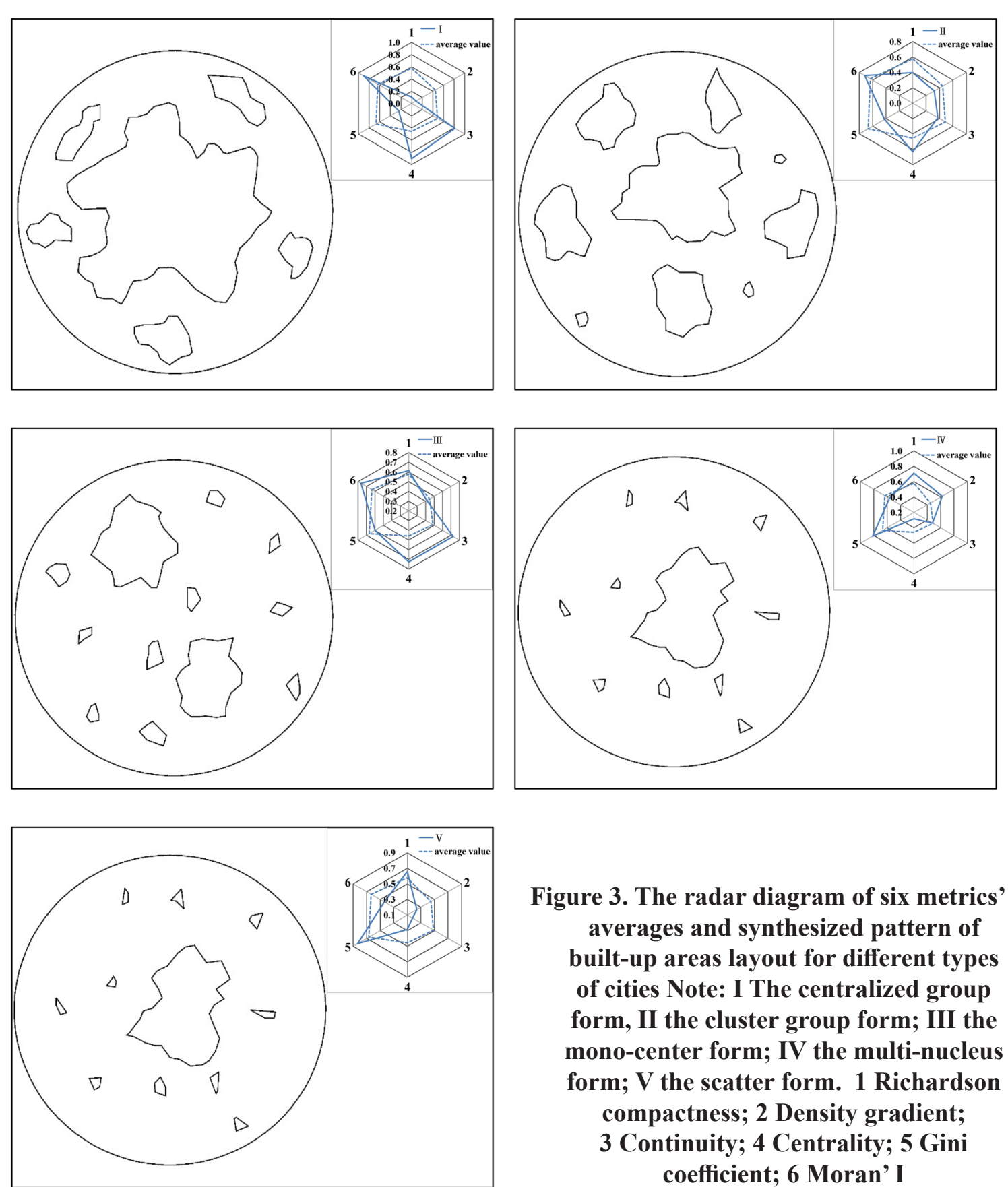

and highly clustered (the high Moran' I), the central area occupies a big proportion (the high continuity), and the peripheral group land shows the trend to the heart (the high centrality). The shape compactness and density gradients are generally low, indicating that the central area is loosely formed. By investigating the real urban cases, the form may be related with the strong radiation effect of big city center of this type of cities, which leads to
Figure 3. The radar diagram of six metrics' averages and synthesized pattern of built-up areas layout for different types of cities Note: I The centralized group form, II the cluster group form; III the mono-center form; IV the multi-nucleus form; $V$ the scatter form. 1 Richardson compactness; 2 Density gradient; 3 Continuity; 4 Centrality; 5 Gini coefficient; 6 Moran' I 
leaping development as well as extension from the center, the surrounding towns expanding to the center, and the overall urban intensity being high.

Type II represents the cities with a cluster group layout, including the three prefecture level cities of Ningbo, Changzhou and Zhenjiang, and the five county-level cities of Yuyao, Cixi, Jiangyin, Changshu, Zhangjiagang and Kunshan. The cities in this type have higher centrality index and Moran' I coefficient, but lower continuity index, indicating that the proportion of the center area is reduced, and the peripheral urban clusters and the center together show the group layout. For the prefecture level cities, due to the more developed new towns and port areas, the central area declines in the dominant position. The county-level city is in the rapid development of industrial parks and township enterprises, which promote a great growth of towns catching up with the central city, accordingly performing a multi-cluster distribution pattern.

Type III represents the mono-center cities, including Nantong, Jiaxing, Yangzhou, Taizhou, Shaoxing five prefecture-level cities and Jingjiang a county-level city. The city type features high continuity index and high centrality index, indicating the prominent position of the central area; at the same time the Moran' I coefficient and Gini coefficient are higher than the mean, indicating the uneven distribution of urban land in the city territory. The central area gathering elements of resources in the polarization stage of development, grows faster than the surrounding and further attracts capital, technology, talent, services and other elements to continuously gather in the center. The gap between center and the external increases and enhances the distribution of single-center urban form.

Type IV is named as the multi-nucleus city form, including one prefecture-level city (Huzhou) and 24 county-level cities, the most of the YRD cities. The Gini coefficient is higher, indicating that the spatial distribution of the urban land is not balanced and concentrated in a few patches. However, the continuity index and the centrality index are lower, revealing that the central area has a low proportion and the other urban areas don't show centripetal agglomeration. In addition, the Moran' I coefficient is lower, indicating that the overall degree of urban clustering is not high, and further reveals the "several dots" layout feature. Investigating the practical cases, the size of the cities belonging to this category is generally small, and the economic activities are concentrated in several towns with outstanding development advantages in the territory, which constitutes the nucleus of urban development. Type $\mathrm{V}$ features a scatter form, including a prefecture-level city of Taizhou and Linhai, Wenling, Fuyang, Shangyu, Jiande five county-level cities. The contiguity index and the Moran' I coefficient of these cities are the lowest among all categories, indicating that the size of the central area is not dominant and the overall degree of agglomeration is low. Gini coefficient is the highest in five categories of cities, showing that the urban development is very uneven. In general, the development of land within the domain is low, and do not have the characteristics of centripetal organization, showing a strong dispersion. Investigating the real scenarios of this kind of cities, the layout of urban development is affected by the natural division of the mountain and the hills as well as the spontaneous construction along roads. The built-up area often appears narrow strips, and the structure of the urban land use is relative loose.

For the above five different types of urban compactness, the biomass loss for urban expansion between 2000-2010 is calculated with the biomass spatial data, and the results show that the average loss of urban type I is the lowest, the average loss of type $\mathrm{V}$ is the highest, the average loss of IV is the second highest, and the average loss of type II is not much different from that of III (Table 3). Based on the morphological characteristics of five types of cities and their biomass loss assessment results, it can be seen that the types with central agglomeration or aggregation around the central group (I, II, III), present the lower biomass loss rate, and accordingly perform better for the ecological efficiency. The development of multi-nucleus and discrete cities is easy to lead to greater biomass loss. This conclusion is consistent with the general understanding of the compact city, and 
reasonable. In order to eliminate the interference of other factors, we calculated the population density and economic density of each type of city, and found that these factors do not have obvious regularity between the various types, and there is no significant correlation with most of the morphological indices. This indicate that the classification results are not affected by these population and economic factors, with independence and stability for featuring urban form. Although the natural conditions such as terrain and vegetation cover influence the spatial distribution of biomass and further influence the biomass loss, the difference existing at the small scale in local area can be neglected considering the average of the city scale. Therefore, we believe that this result can be used to guide the urban planning in the study area. In order to maximize the effects of the compactness, the city should choose a centripetal and clustering structure: for the city with a stable dominant center, the new clusters should be located around; for the small and medium-sized cities at the fast growth stage without a strong center, strengthen and expand the central area is conducive to the formation of a compact and efficient form.

\section{Conclusion}

In the Yangtze River Delta, where the urban population density is high, the urban spatial compactness is diverse, involving the geometric compactness of the central city, the close relationship between the center and the periphery, and the global agglomeration. Five different types of representations are defined as the basis for the performance assessment and planning of urban spatial structure. The analysis manifests that the compact structure of centripetal gather can play a better ecological benefit. For the small and mediumsized cities which are in the stage of growth and not forming a strong center, it is beneficial to strengthen and expand the central group to form a compact and efficient form structure. The current study only takes this dimension of biomass to account for the stability and significance of the compactness classification, which should be used for more evaluation of the effects of urban morphology.

\section{References}

Angel, S., Sheppard, S. C. \& Civco, D. L. (2005) The dynamics of global urban expansion. Work report by Transport and Urban Development Department, World Bank, Washington, DC.

Brueckner, J. K., Fansler, D. A. (1983) 'The economics of urban sprawl: theory and evidence on the spatial sizes of cities', J. Rev. Econ. Stat. 65(3), 479-482.

Chen, S., Yao, S. \& Wu, J. (2009) 'Policy instrument of urban growth management and its effectiveness in Nanjing', Acta Geographica Sinica, 64 (4), 487-497 (in Chinese).

Cole, J. P., (1964) 'Study of major and minor civil divisions in political geography', University of Nottingham, Mimeograph (presented to the 20th international Geographical Congress, Sheffield).

Burton, E. (2002) 'Measuring urban compactness in UK towns and cities', Environment and Planning B: Planning and Design, 29 (2), 219-250.

Fang, C. \& Qi, W. (2007) 'Research progress and thinking of compact city and its measurement methods', Urban Planning Forum (4), 65-73 (in Chinese).

Galster, G., Hanson, R., Ratcliffe, M. R., Wolman, H., Coleman, S. \& Freihage, J. (2001) 'Wrestling sprawl to the ground: Defining and measuring an elusive concept', Housing Policy Debate, 12, 681-717.

Li, G.-Y., Chen, S., Zhang, H. \& Zhang, T. (2016) 'Variation of spatial estimation and distribution of vegetation biomass in Yangtze River Delta during 2000-2010', Journal of Ecology and Rural Environment, 32 (5), 708-715 (in Chinese).

Ma, L. \& Jin, F. (2011) 'Evaluation of Chinese urban compactness', Progress in Geography, 30 (8), 1014-1020. (in Chinese)

Hagen-Zanker, A. \& Timmermans, H. (2008) 'A Metric of Compactness of Urban Change Illustrated to 22 European Countries', in L. Bernard, A. Friis-Christensen \& H. Pundt (eds.), The European Information Society: 
Taking Geoinformation Science One Step Further (pp. 181-200), Berlin, Heidelberg: Springer Berlin Heidelberg.

Huang, J., Lu, X. \& Sellers, J. M. (2007) 'A global comparative analysis of urban form: Applying spatial metrics and remote sensing', Landscape and Urban Planning, 82 (4), 184-197.

Richardson, H. W. (1973) The economics of urban size, Saxon House, Mass.

Silva, M. (2016) 'Urban morphology and energy: progress and prospects', Urban Morphology, 20 (1), 72.

Tsai, Y. H. (2005) 'Quantifying Urban Form: Compactness versus "Sprawl", Urban Studies, 42 (1), 141-161 